\title{
LAS TERMITAS COMO AGENTES NATURALES DE ALTERACIONES TAFONÓMICAS POSTDEPOSICIONALES EN UN ESQUELETO HUMANO DEL SITIO ARQUEOLÓGICO TOCA DO ENOQUE (PIAUÍ, BRASIL)
}

\author{
TERMITES AS NATURAL AGENTS OF POSTDEPOSITIONAL TAPHONOMIC \\ ALTERATIONS IN A HUMAN SKELETON FROM TOCA DO ENOQUE \\ ARCHAEOLOGICAL SITE (PIAUÍ, BRAZIL)
}

\author{
Ana Solari ${ }^{*}$, Anne Marie Pessis ${ }^{1,2}$, Gabriela Martinn ${ }^{1,2}$, Fátima Barbosa ${ }^{2,3}$ y Sérgio F.S. Monteiro \\ da Silva ${ }^{1}$ \\ ${ }^{I}$ Programa de Pós-Graduação em Arqueologia. Universidade Federal de Pernambuco (UFPE). Recife. Brasil \\ ${ }^{2}$ Fundação Museu do Homem Americano (FUMDHAM). Piauí. Brasil \\ ${ }^{3}$ Colegiado de Arqueologia e Preservação Patrimonial. Universidade Federal do Vale do São Francisco (UNIVASF). Piauí. Brasil
}

PALABRAS CLAVE: tafonomía; icnoarqueología; arqueoentomología funeraria; isópteros; región Neotropical

RESUMEN Las termitas son insectos que aparecen muchas veces en contextos arqueológicos en Brasil y casi nunca son objeto de estudio como procesos naturales de formación de los depósitos y contextos arqueológicos. La probabilidad de que se encuentren marcas de estos insectos modificadores de carcasas de animales y humanos en sitios arqueológicos en Brasil y en Sudamérica hace pertinente su estudio. A partir de una aproximación teórica - metodológica que comprende la Icnoarqueología y la Arqueoentomología Funeraria, presentamos el caso de estudio de un esqueleto humano con señales de bioerosión debidas a la acción de termitas subterráneas en el sitio arqueo- lógico Toca do Enoque (Piauí, nordeste de Brasil) del Holoceno Medio. Guiados por un protocolo de estudio actualístico, pudimos registrar que el patrón de daños observado en los huesos resultó ser consistente con el comportamiento osteofágico de ciertas especies de termitas endémicas de la región Neotropical (Familia: Termitidae). El análisis macroscópico muestra la importancia de reconocer a los isópteros subterráneos como agentes tafonómicos naturales que pueden actuar en la degradación postdeposicional de restos óseos humanos provenientes de contextos funerarios arqueológicos. Rev Arg Antrop Biol 22(1), 2020. doi: $10.24215 / 18536387 \mathrm{e} 016$

KEYWORDS taphonomy; icnoarchaeology; funerary archeoentomology; termites; Neotropical region.

ABSTRACT Termites are insects that often appear in archaeological contexts in Brazil, that are almost never studied as natural processes of deposit formation and archaeological contexts. The probability of founding marks of these insects that modify carcasses of animals and humans in archaeological sites in Brazil and South America makes their study relevant. From a theoretical - methodological approach that includes Icnoarchaeology and Funeral Archeoentomology, we present the case study of a human skeleton with signs of bioerosion due to the action of subterranean termites in the archaeological site at
Toca do Enoque (Piauí, northeastern Brazil) from the Middle Holocene. Guided by an actualist study protocol, we were able to record that the pattern of damage observed in the bones was consistent with the osteophageal behaviour of certain termite species endemic to the neotropical region (Family: Termitidae). The macroscopic analysis shows the importance of recognizing subterranean termites as natural taphonomic agents that act in postdepositional degradation of human bone remains from archaeological burial contexts. Rev Arg Antrop Biol 22(1), 2020. doi:10.24215/18536387e016
A pesar de que el rol de los insectos necrófagos es bien conocido en las ciencias forenses, su participación como agentes tafonómicos de degradación ósea es menos conocida (Queiroz et al., 2017). Sin embargo, diferentes órdenes de artrópodos pueden ser responsables de una bioerosión significativa en huesos humanos y de fauna (Matthew, 2018). La identificación e interpretación de dichas lesiones son importantes para la comprensión de los fenómenos tafonómicos naturales postdeposicionales actuantes en un de-
Financiamiento: Fundação Museu do Homem Americano (FUMDHAM). Beca CAPES-PNPD otorgada a Ana Solari.

\footnotetext{
*Correspondencia a: Ana Solari. Departamento de Arqueologia. Centro de Filosofia e Ciências Humanas. $10^{\circ}$ andar Av. da Arquitetura S/N . PE - CEP: 50740-550. Cidade Universitária. Recife. Brasil.

E-mail: anasolari74@gmail.com
}

Recibido 18 Febrero 2019; aceptado 30 Mayo 2019

doi:10.24215/18536387e 016 
pósito funerario y en el entendimiento de los procesos de formación del registro bioarqueológico.

En Brasil están presentes cerca de 300 especies integrantes de cuatro de las siete familias del orden Isóptera: Serritermitidae, Rhinotermitidae, Kalotermitidae y Termitidae. La familia Termitidae es la que contiene el mayor número de especies conocidas y de mayor representatividad en Brasil (85\%) (Constantino, 1999; Fontes, 1995; Lima y Costa-Leonardo, 2007). En particular, en las proximidades a nuestra área de estudio, en el Parque Nacional Serra da Capivara (Piauí), han sido investigados los principales efectos de la bioturbación producida por termitas de la familia Termitidae y del género Nasutitermes en los paredones rocosos con arte rupestre (Silva y Andrade, 2016), sin embargo, en nuestro caso nos interesa mostrar los efectos de estos agentes tafonómicos sobre los restos óseos humanos dado sus implicaciones para la interpretación de los contextos mortuorios.

Distinguir correctamente los procesos y agentes de modificación postmortem sobre los huesos humanos resulta fundamental para entender los procesos de formación del registro arqueológico e interpretar adecuadamente los contextos funerarios. Para eso, presentamos el caso de estudio de un esqueleto humano del Entierro 3 de Toca do Enoque, un sitio arqueológico datado en el Holoceno Medio y localizado en el Parque Nacional Serra das Confusões (Piauí). Por medio de una aproximación teórica - metodológica que envuelve la Icnoarqueología (Baucon et al., 2008) y la Arqueoentomología Funeraria (Huchet, 2014a, 2014b), pudimos registrar una serie de señales de bioerosión observables macroscópicamente y reconocer un patrón de daños en el esqueleto compatibles con la actividad de insectos, específicamente por termitas subterráneas de la familia Termitidae, endémicas de la región neotropical del nordeste brasilero (Thorne y Kimsey, 1983).

\section{UNA APROXIMACIÓN AL ESTUDIO CIENTÍFICO DE LOS INSECTOS Y SU APORTE AL CONOCIMIENTO DEL PASADO HUMANO}

El estudio científico de los restos de insectos en contextos arqueológicos se inicia con la Paleo-entomología (Buckland y Coope, 1991), la Arqueo-entomología (Moret, 1996) o la Arqueoentomología Funeraria (Huchet, 1996, 2014a, 2014b). Dichas disciplinas se dedican a la aplicación de la entomología al estudio de las sepulturas y los restos humanos de contextos arqueológicos, permitiendo contribuir a la resolución de problemas arqueológicos y bioarqueológicos que involucren la interacción entre las sociedades del pasado, su ambiente y la dinámica biodiversidad de insectos (Vanin y Huchet, 2017).

Al mismo tiempo, la presencia de insectos dentro de las sepulturas se puede revelar por las huellas de actividad dejadas en los huesos u otros materiales. El análisis, descripción e interpretación de dichas huellas forman parte de una disciplina que estudia la interacción entre el organismo y el sustrato en el cual se conserva la impronta. El estudio de las huellas o marcas dejados por dichos agentes en los restos óseos humanos o animales se enmarca en la Icnología (Bertling et al., 2006; Frey, 1973) que se ocupa del análisis y descripción de las señales de actividad dejadas por un organismo en el curso de su vida. La aplicación de los principios y métodos de esa disciplina en contextos arqueológicos se conoce como Icnoarqueología (Baucon et al., 2008).

En un contexto funerario, las huellas en el tejido óseo resultan de dos procesos diferentes (no excluyentes entre sí) de naturaleza mecánica o química, como resultado de procesos pre y post - deposicionales producto de las actividades de diferentes hexápodos. Esos dos tipos de bioerosiones producen daños notables en los esqueletos, por lo cual su reconocimiento y caracterización resultan fundamentales para los bioarqueólogos. La identificación taxonómica de los agentes bióticos responsables de las degradaciones óseas documenta sobre el ambiente postdeposicional del cadáver en su lugar de depósito. La identificación del agente responsable de las lesiones y su relación con los huesos alterados permiten una aproximación a la historia tafonómica del conjunto óseo. Así, las marcas de actividad relativas al comportamiento osteofágico de ciertos insectos, pueden proveer informaciones sobre las practicas funerarias del pasado (Huchet, 2014a, 2014b; Matthew, 2018). 


\section{Las termitas como agentes tafonómicos de degradación ósea}

Los insectos necrófagos, principalmente de las órdenes dípteros y coleópteros, son bien conocidos como agentes responsables del proceso de descomposición y esqueletización de un cadáver, pero es menos conocido el rol de los insectos como agentes tafonómicos de degradación del tejido óseo en contextos arqueológicos, especialmente de los artrópodos (Byrd y Castner, 2001; Huchet, Deverly, Gutierrez y Chauchat, 2011; Nystrom, Goff y Goff, 2005; Smith, 1986; Watson y Abbey, 1986).

Entre los agentes tafonómicos no humanos que actúan en la degradación ósea, se encuentran diferentes órdenes de artrópodos, incluyendo los isópteros. Los isópteros son una de las órdenes de insectos capaces de producir señales en los huesos, además de explotar, consumir y alterar los restos óseos (Huchet, 2014a, 2014b).

Generalmente se asume que las termitas son principalmente insectos xilófagos consumidores de madera y celulosa (Grassé, 1982; Miller, 2010), sin embargo, ha sido demostrado que una gran variedad de materiales orgánicos en diversos grados de descomposición sirve de alimento para estos insectos, incluyendo madera (viva o muerta), gramíneas, plantas herbáceas, hongos, líquenes, humus, nidos de otras especies de termitas, excrementos y carcasas de animales (Lima y Costa-Leonardo, 2007; Wood, 1978).

Las deficiencias nutricionales por falta de nitrógeno en la dieta xilófaga basada en celulosa, especialmente en condiciones estacionales de sequía, serían la principal causa de la actividad osteofágica de algunas especies de termitas (Lima y Costa-Leonardo, 2007; Prestes, Tepedino, Kosmann y Pujol-Luz, 2014; Prestwich, Bentley y Carpenter, 1980; Slaytor y Chappell, 1994). En determinadas condiciones, las termitas subterráneas son capaces de consumir tejido óseo en cualquier estado de preservación, desde hueso fresco hasta altamente degradado, dejando una serie de señales características de tales actividades osteofágicas en los restos óseos consumidos (Thorne y Kimsey, 1983).
En particular, tres familias de termitas (Termitidae, Mastotermitidae y Rhinotermitidae) han sido reconocidas por su habilidad de consumir huesos, su impacto en la degradación de restos óseos ha sido demostrada desde hace por lo menos un siglo y sus huellas diagnosticas identificadas más recientemente (Backwell, Parkinson, Roberts, D'Errico y Huchet, 2012; Huchet, 2014a, 2014b; Huchet et al., 2011; Matu, Crevecoeur y Huchet, 2017).

A partir del trabajo pionero de Derry (1911) que identificó a las termitas como agentes tafonómicos de modificación ósea en tumbas con momias de la antigua Nubia, otros casos arqueológicos involucrando diversas clases de insectos y su acción en restos humanos han sido descritos y publicados desde entonces. Entre ellos, están los análisis de huesos fósiles con marcas de termitas en sitios arqueológicos y paleoantropológicos africanos (Kaiser, 2000; Matu et al., 2017; McBrearty, 1990), el análisis de la fauna cadavérica en una momia egipcia (Huchet, 2010) y en un fardo funerario del México prehispánico (Huchet et al., 2013), la aplicación de la entomología forense para evaluar el intervalo postmortem en el caso de una momia Chachapoyas en Perú (Nystrom et $a l ., 2005)$, o el primer caso arqueológico en el continente sudamericano de un esqueleto afectado por termitas en el sitio Huaca de la Luna en Perú (Huchet et al., 2011). En varios de estos trabajos, se han aplicado protocolos de reconocimiento de las huellas dejadas por las termitas a partir de modificaciones óseas diagnósticas de acuerdo con una aproximación multidisciplinar que involucra la Icnología, la Tafonomía, la Entomología, la Arqueología y la Bioarqueología.

\section{MATERIAL Y MÉTODOS}

El sitio Toca do Enoque (Faure, Guérin y Luz, 2011; Guidon y Luz, 2009; Kinoshita et al., 2016; Luz, 2014) es un abrigo rocoso localizado en la Serra das Andorinhas en el Parque Nacional Serra das Confusões, sudoeste del estado de Piauí, en las coordenadas $43^{\circ} 55^{\prime} 625^{\prime}$ " longitud O y 9 $9^{\circ} 14 ' 653^{\prime}$ " latitud $\mathrm{S}$ (Fig. 1). Sus medidas son de $60 \mathrm{~m}$ de largo 
y $10 \mathrm{~m}$ de altura, y está orientado en sentido noroeste-sudeste con la abertura hacia el sudoeste. En su paredón fueron representados grafismos rupestres geométricos y zoomorfos de las tradiciones Nordeste, Agreste y Geométrica (Pessis, 1992).

Las excavaciones se llevaron a cabo entre 2008 y 2009 por la arqueóloga fallecida Fátima Luz con la coordinación de Niede Guidon y la colaboración de un equipo de la Fundação do Museu do Homem Americano (FUMDHAM), y dieron como resultado el hallazgo de tres entierros. El Entierro 1, individual primario, el Entierro 2, múltiple primario y secundario, y el Entierro 3, individual primario perturbado natural y antrópicamente, foco del presente trabajo.

Cronológicamente, el uso funerario del sitio fue estimado a través de las dataciones indirectas sobre carbones asociados que dieron una antigüedad de 5930 \pm 50 años AP (BETA252374) para el Entierro 1; entre $6220 \pm 50$ a $6610 \pm 40$ años AP (BETA-257093 y BETA257092) para el Entierro 2; y $3430 \pm 40$ años
AP (BETA-252607) para el Entierro 3 (Faure et al., 2011; Guidon y Luz, 2009; Kinoshita et al., 2016; Luz, 2014). La ausencia de colágeno en huesos humanos y animales del sitio han imposibilitado conseguir dataciones directas. Una descripción detallada de las excavaciones de los tres entierros, las primeras interpretaciones, los estudios sobre sus acompañamientos funerarios, o sobre la datación de sus materiales se pueden consultar en los trabajos de Faure et al. (2011), Guidon y Luz (2009), Kinoshita et al. (2016) y en la tesis de doctorado de Fátima Luz (Luz, 2014).

En particular, el Entierro 3 (Fig. 2) puede caracterizarse como un entierro individual primario con perturbaciones tafonómicas postdeposicionales culturales y naturales, cuyo contexto original, posición y orientación del cuerpo son parcialmente desconocidas (Duday, Courtaud, Crubezy, Sellier, y Tillier, 1989; Sprague, 2005). Al momento de la excavación (Luz, 2014) pudo verificarse una fosa de formato ovalada y coloración oscura producto

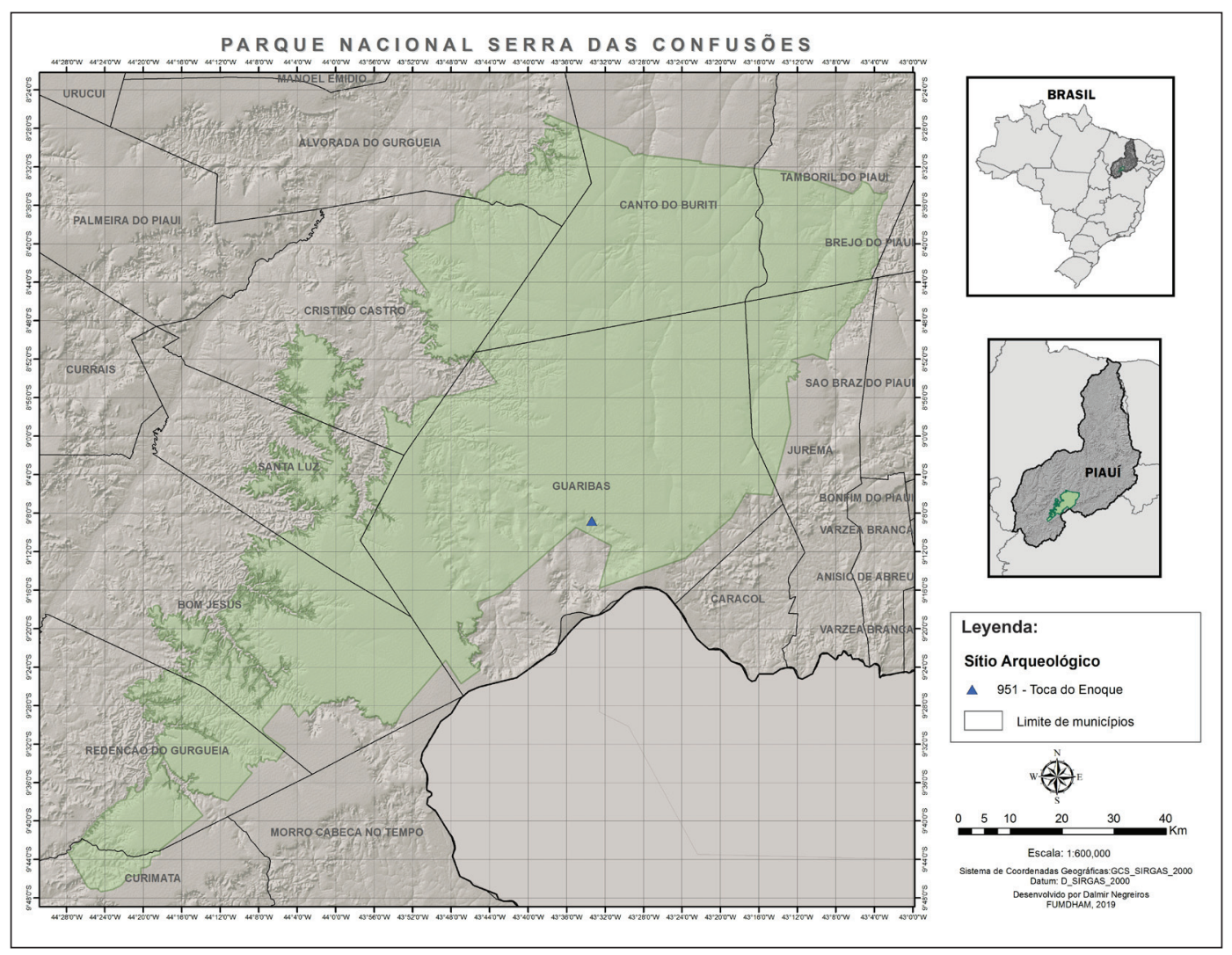

Fig. 1. Mapa con la localización del sitio arqueológico Toca do Enoque, Piauí, Brasil. 


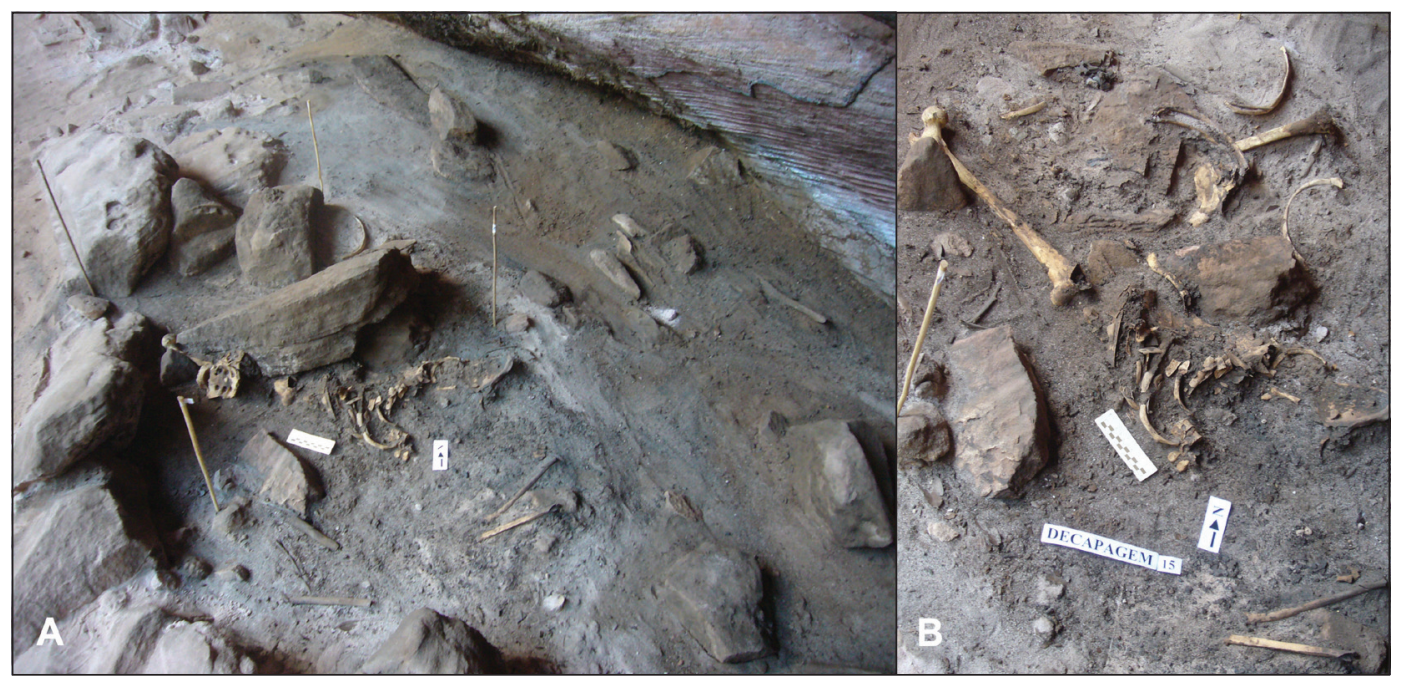

Fig. 2. Entierro 3 de Toca do Enoque. A: Vista general. B: Detalle.

de los abundantes restos vegetales (hojas, semillas, madera) que contenía, rodeada por una estructura de bloques de piedra, donde estaban depositados los restos óseos humanos dispersos y sin conexión anatómica de un esqueleto incompleto pertenecientes a un individuo adulto (menor de 40 años) y sexo masculino (Bass, 1987; Brothwell, 1987; Buikstra y Ubelaker, 1994; Ubelaker, 1978; White y Folkens, 2000). En partes de la fosa fueron percibidas áreas intencionalmente quemadas, restos de hogueras, abundantes carbones y cenizas, junto con algunos huesos del esqueleto parcialmente quemados. También fueron observados nidos de termitas en el área de la sepultura y grandes bloques de piedra entre los restos óseos producto de la perturbación postdeposicional (Luz, 2014). Al tratarse de un entierro perturbado, la datación indirecta obtenida para este individuo de $3430 \pm 40$ años AP (BETA-252607), que se distancia notablemente en relación a los otros dos entierros del sitio y sitúan el uso funerario del abrigo alrededor de 6000 años antes del presente, podría en realidad estar vinculada al momento de alteración antrópica de la sepultura donde los huesos fueron intencionalmente desordenados, algunos parcialmente quemados y muchos de ellos removidos completamente de la fosa (Fig. 3).

La participación de las termitas como agentes tafonómicos naturales de perturbación postdeposicional del Entierro 3, fue sugerida en campo ante la presencia de nidos de termitas en el área de la fosa.
Para verificar dicha participación, posteriormente en laboratorio fueron observados macroscópicamente los huesos humanos del esqueleto aplicando los protocolos para el reconocimiento de las actividades de termitas subterráneas sobre el tejido óseo de Backwell et al. (2012) y Huchet (2014a, 2014b).

Al respecto, para identificar la actividad osteofágica de las termitas sobre los restos óseos, Backwell et al. (2012) establecieron un protocolo de ocho modificaciones óseas diagnósticas a partir de un experimento actualístico basado en el estudio macroscópico y microscópico de los huesos (Tabla 1). Estas van desde la presencia de un residuo oscuro producido por las termitas que se adhiere firmemente a las superficies óseas, hasta la destrucción parcial o total del tejido óseo (especialmente el tejido esponjoso), e incluyen entre ambos extremos de daños al hueso otras señales de alteraciones tafonómicas como hoyos superficiales y profundos, marcas en forma de estrella, textura de la superficie grabada, o estriaciones paralelas y sub-paralelas (Backwell et al., 2012:79). Además, según Huchet (2014a, 2014b), la presencia de termitas también puede reconocerse por el hallazgo de restos de galerías-túneles de termitas asociadas a los restos óseos (estructuras tubulares, mezcla de tierra, saliva y excrementos por donde se desplazan las termitas, extremadamente sensibles a la desecación) y por las huellas que dichas estructuras dejan cuando se adhieren fuertemente a la superficie ósea cortical aun cuando no produzcan señales de daños evidentes. 


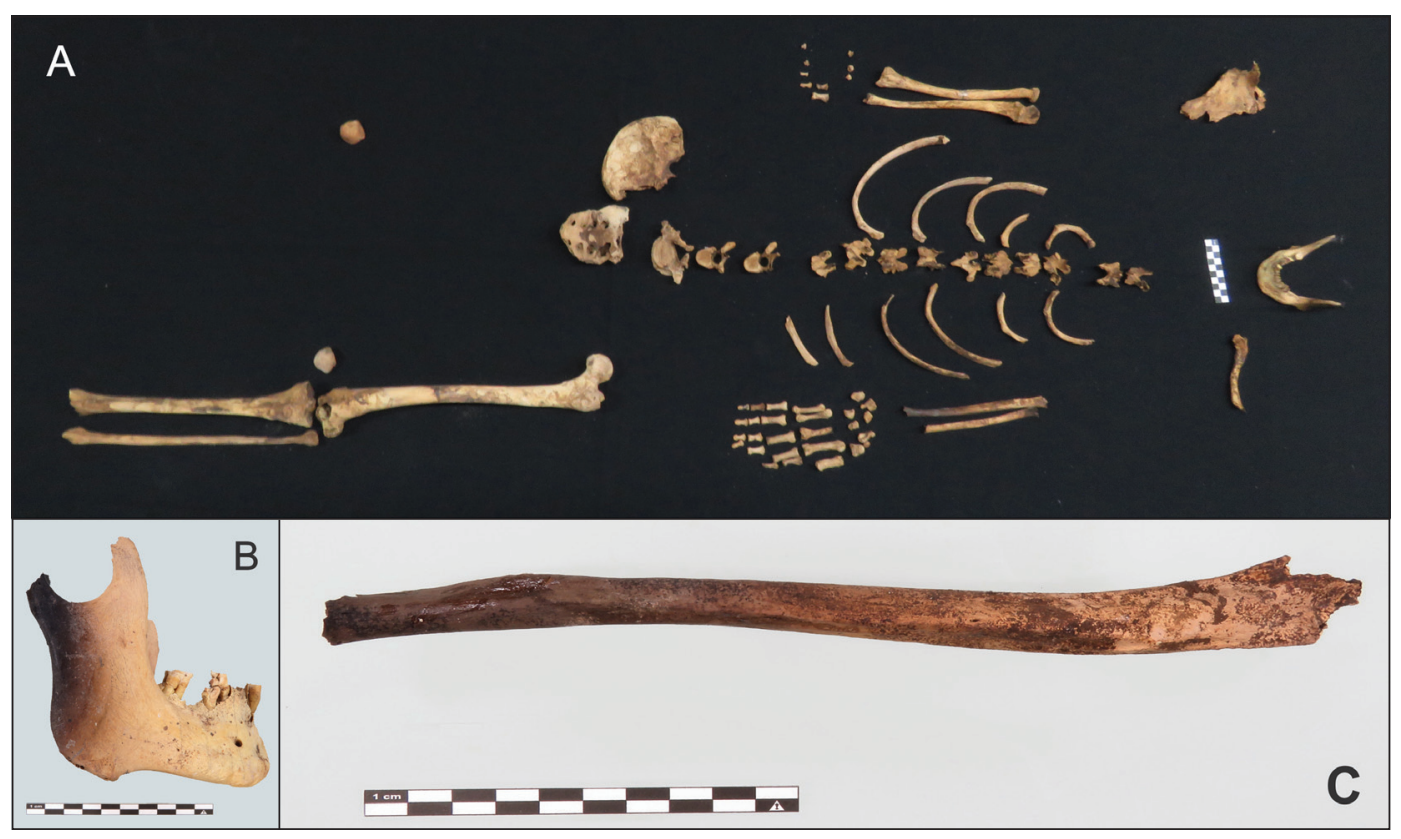

Fig. 3. A: Esqueleto preservado del Entierro 3 de Toca do Enoque (los huesos ausentes habrían sido retirados intencionalmente durante la perturbación postdeposicional) (Escala: 10cm). B: Mandíbula parcialmente quemados (Escala: $10 \mathrm{~cm})$. C: Cúbito parcialmente quemado (Escala: $10 \mathrm{~cm})$.

TABLA 1: Tipos de modificación ósea causada por termitas según el protocolo experimental de Backwell et al. 2012 (Traducido y resumido de la Tabla 2, p. 79)

\begin{tabular}{|c|c|c|}
\hline $\begin{array}{c}\text { Tipos de } \\
\text { modificación ósea }\end{array}$ & Descripción & $\begin{array}{c}\text { Nivel de } \\
\text { observación }\end{array}$ \\
\hline Destrucción & Obliteración del hueso & $\begin{array}{l}\text { Visibilidad } \\
\text { macroscópica }\end{array}$ \\
\hline $\begin{array}{l}\text { Agujeros } \\
\text { profundos }\end{array}$ & $\begin{array}{c}\text { Agujeros semicirculares que penetran la lámina cortical externa y/o } \\
\text { el hueso esponjoso hasta la cavidad medular }\end{array}$ & $\begin{array}{l}\text { Visibilidad } \\
\text { macroscópica }\end{array}$ \\
\hline $\begin{array}{l}\text { Aspecto } \\
\text { grabado }\end{array}$ & $\begin{array}{c}\text { Remoción de las laminillas externas para exponer la estructura } \\
\text { ósea subyacente }\end{array}$ & $\begin{array}{l}\text { Visibilidad } \\
\text { macroscópica }\end{array}$ \\
\hline $\begin{array}{l}\text { Residuo de } \\
\text { superficie }\end{array}$ & $\begin{array}{l}\text { Recubrimiento de color marrón oscuro a negro que decolora el hueso y se } \\
\text { asocia con la destrucción y el grabado de la superficie del hueso }\end{array}$ & $\begin{array}{l}\text { Visibilidad } \\
\text { macroscópica }\end{array}$ \\
\hline $\begin{array}{l}\text { Hoyos } \\
\text { superficiales }\end{array}$ & $\begin{array}{l}\text { Depresiones semicirculares anidadas en el hueso que muestran } \\
\text { bordes roídos dispuestos radialmente y comprenden } \\
\text { superposiciones individuales superpuestas y paralelas }\end{array}$ & $\begin{array}{l}\text { Visibilidad } \\
\text { intermedia }\end{array}$ \\
\hline $\begin{array}{l}\text { Marcas de } \\
\text { forma } \\
\text { estrellada }\end{array}$ & $\begin{array}{l}\text { Numerosas, y a veces superpuestas, estrías / surcos individuales } \\
\text { radialmente organizadas alrededor de una cavidad con paredes } \\
\text { lisas. Las estrías que constituyen las marcas en forma de estrella } \\
\text { tienen un perfil en forma de U y una morfología interna suave. }\end{array}$ & $\begin{array}{l}\text { Visibilidad } \\
\text { intermedia }\end{array}$ \\
\hline $\begin{array}{c}\text { Estrías } \\
\text { paralelas }\end{array}$ & $\begin{array}{l}\text { Múltiples estriaciones relativamente paralelas ubicadas a lo largo y orientadas } \\
\text { perpendiculares a bordes o extremos rotos. También se conocen como bordes } \\
\text { roídos. Tienen un perfil en forma de U y una suave morfología interna. }\end{array}$ & $\begin{array}{l}\text { Visibilidad } \\
\text { microscópica }\end{array}$ \\
\hline $\begin{array}{c}\text { Estrías } \\
\text { subparalelas }\end{array}$ & $\begin{array}{l}\text { Agrupaciones de estrías subparalelas orientadas al azar ubicadas } \\
\text { principalmente en el periostio o cuerpo de los especímenes, lejos } \\
\text { de los bordes. Tienen un perfil en forma de U y una suave morfología interna. }\end{array}$ & $\begin{array}{l}\text { Visibilidad } \\
\text { microscópica }\end{array}$ \\
\hline
\end{tabular}




\section{RESULTADOS}

A partir de la aplicación de los protocolos de Backwell et al. (2012) y Huchet (2014a, 2014b), fueron observadas sobre los huesos del esqueleto del Entierro 3, señales de bioerosión por acción mecánica y/o química indicando indudablemente la acción de termitas subterráneas, posiblemente de la familia Termitidae endémica de la región Neotropical en la que se localiza el sitio arqueológico (Thorne y Kimsey, 1983). Prácticamente todos los huesos conservados del esqueleto del Entierro 3 de Toca do Enoque, presentaron una o más modificaciones óseas vinculadas a la acción de termitas. En- tre las principales señales observadas, registramos la presencia de los restos de galerías y/o túneles, y las huellas superficiales que dichas galerías y/o túneles dejaron marcadas sobre las superficies óseas, así como la presencia del residuo oscuro producido por las termitas que resultó adherido firmemente a la superficie cortical. Asimismo, fueron vistas algunas señales de hoyos o perforaciones superficiales y un poco más profundas, y también la destrucción parcial o total de ciertos segmentos óseos, especialmente en áreas de tejido óseo esponjoso, principalmente en vértebras, costillas y epífisis de huesos largos. La Figura 4, muestra un ejemplo de cada caso observado.

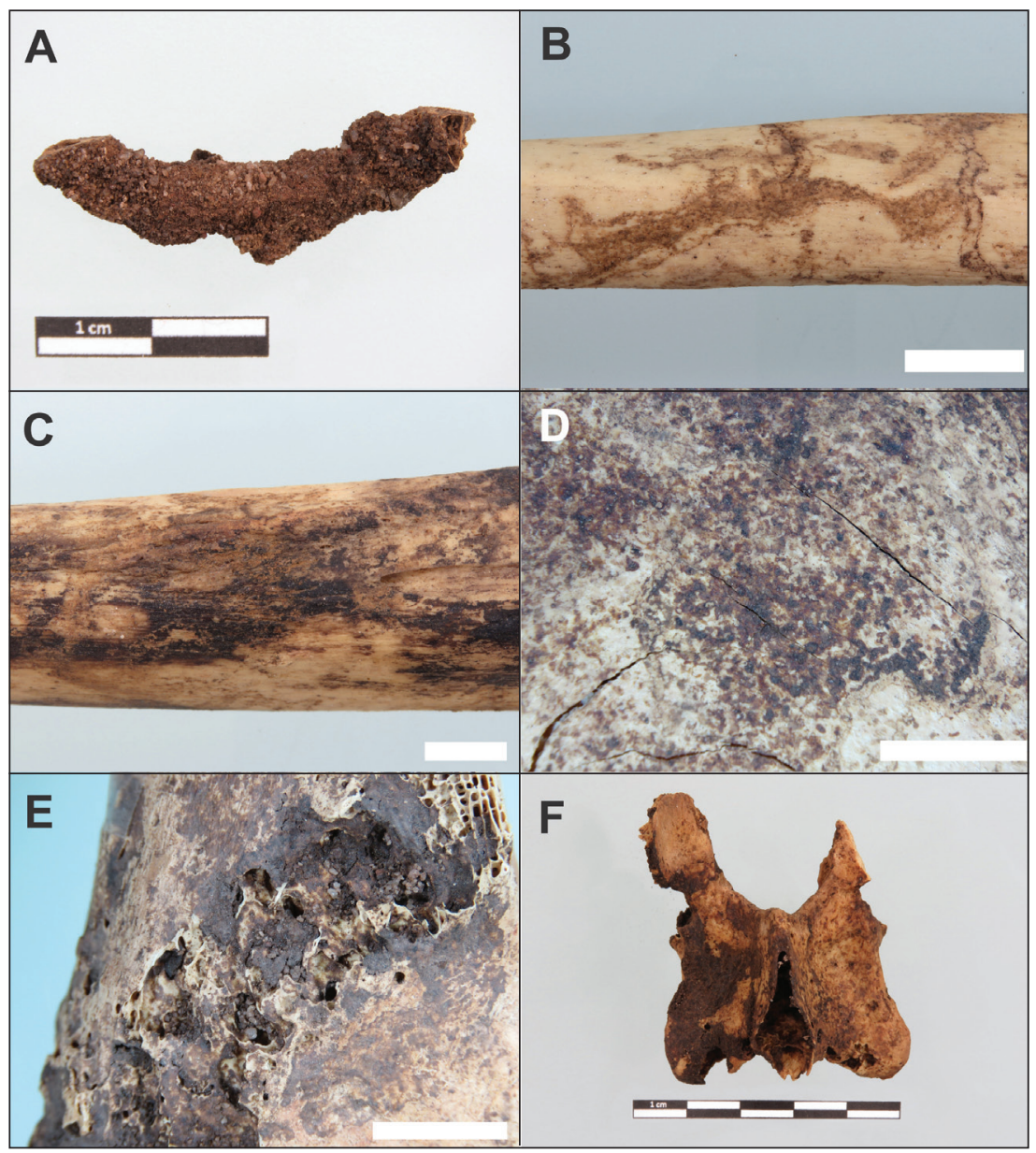

Fig. 4. Señales de bioerosión por acción mecánica y/o química indicando la acción de termitas subterráneas. A: Restos de galerías y/o túneles (Escala: $2 \mathrm{~cm}$ ). B: Huellas superficiales que las galerías y/o túneles dejaron marcadas sobre las superficies óseas (Escala: $1 \mathrm{~cm}$ ). C: Presencia del residuo oscuro producido por las termitas que resultó adherido firmemente a la superficie cortical (Escala: $1 \mathrm{~cm})$. D: Hoyos o perforaciones superficiales (Escala: $1 \mathrm{~cm})$. E: Hoyos o perforaciones profundas (Escala: $1 \mathrm{~cm}$ ). F: Destrucción parcial o total de segmentos óseos, especialmente en áreas de tejido óseo esponjoso (Escala: $5 \mathrm{~cm}$ ). 
Así, consideramos que la colonización postdeposicional del Entierro 3 por termitas subterráneas endémicas de la región, se debió haber motivado principalmente por la cantidad de componentes vegetales (abundantes hojas, semillas y maderas), que seguramente componían la estructura funeraria original y que resultaron atractivos para la dieta xilófaga de la colonia de isópteros. A pesar de la perturbación antrópica postdeposicional, esos componentes vegetales forman parte de las otras dos estructuras funerarias no perturbadas del sitio y ciertamente también formaban parte de este. Con lo cual, el consumo parcial del esqueleto pudo haber sido una consecuencia secundaria de dicha colonización. Las deficiencias nutricionales motivadas por la estacionalidad y el clima en periodo de sequía, debieron ser otros factores condicionantes para la colonización del entierro por termitas y su comportamiento osteofágico (Huchet et al., 2011; Vanin y Huchet, 2017)Finalmente, aunque fueron contemplados otros procesos y agentes tafonómicos naturales y culturales en los daños observados sobre el esqueleto del Entierro 3, incluyendo el $\mathrm{pH}$ del sedimento (Gordon y Buikstra, 1981) o la perturbación antrópica (Weiss-Krejci, 2011) que removió, mezcló e incluso quemó ligeramente algunos huesos, el foco principal de este trabajo está centrado en la acción de las termitas como agentes tafonómicos de perturbación natural postdeposicional del esqueleto.

\section{CONCLUSIONES}

Los isópteros de la familia Termitidae, endémicos de la región Neotropical del nordeste brasilero, son agentes potenciales de perturbación tafonómica postdeposicional en sitios arqueológicos y sus actividades pueden afectar a la interpretación de los procesos de formación del sitio. En particular, el comportamiento osteofágico y su consecuente destrucción ósea son importantes procesos tafonómicos que influyen en la interpretación de los contextos mortuorios, sobre todo, en el caso de entierros perturbados como el Entierro 3 de Toca do Enoque (Prestes et al., 2014; Queiroz et al., 2017; Watson y Abbey, 1986). Entender la acción simultánea y/o sucesiva de los agentes y procesos tafonómicos naturales y culturales que actuaron en la formación de los depósitos arqueológicos de tipo funerario es fundamental para interpretar correctamente las prácticas mortuorias del pasado y las perturbaciones postdeposicionales que potencialmente hayan sufrido desde el momento del depósito del cadáver hasta su descubrimiento en el presente.

La combinación entre una aproximación teórica-metodológica desde la Arqueoentomología Funeraria y la Icnoarqueología, permiten un mejor entendimiento e interpretación de los procesos tafonómicos postdeposicionales para la reconstrucción de las practicas funerarias del pasado. A partir de la literatura especializada consultada, que describe la morfología de las señales de daño causadas por insectos como las termitas, podemos concluir que estos fueron los agentes tafonómicos naturales responsables de algunos de los principales daños observables en el esqueleto del Entierro 3 de Toca do Enoque. La mayoría de los huesos analizados del esqueleto del Entierro 3 de Toca do Enoque mostraron algunas de las modificaciones superficiales observables macroscópicamente de acuerdo con el protocolo de daños propuesto por Backwell et al. (2012) y Huchet (2014a, 2014b) incluyendo: destrucción parcial o total de segmentos óseos (especialmente en vértebras, costillas y epífisis de huesos largos), textura de la superficie ósea grabada, presencia de un residuo de superficie oscuro y marcas superficiales de las galerías-túneles.

Teniendo en cuenta la amplia representación y distribución de isópteros en la región Neotropical y el reconocimiento de las actividades osteofágicas de varias familias de termitas endémicas, el análisis de sus huellas diagnósticas debería ser mejor estudiado en contextos arqueológicos potencialmente afectados por dichos agentes tafonómicos. Aunque se trate de un estudio de caso, esperamos que este trabajo muestre la importancia de avanzar sobre este tipo de estudios tafonómicos para una adecuada interpretación de las prácticas funerarias, fundamentalmente en contextos mortuorios perturbados que indican la participación de varios agentes y/o procesos tafonómicos postdeposicionales naturales y/o culturales simultáneos o consecutivos en la formación del registro arqueológico. 


\section{AGRADECIMIENTOS}

In Memoriam Dra. Fátima Luz. Los autores agradecen la contribución del equipo técnico profesional de la FUMDHAM que participaron en las actividades de campo y laboratorio, y especialmente a la Dra. Niede Guidon.

\section{LITERATURA CITADA}

Backwell, L. R., Parkinson, A. H., Roberts, E. M., D’Errico, F. y Huchet, J. B. (2012). Criteria for identifying bone modification by termites in the fossil record. Palaeogeography, Palaeoclimatology, Palaeoecology, 337 338, 72-87. doi:10.1016/j.palaeo.2012.03.032

Baucon, A., Privitera, S., Morandi Bonacossi, D., Canci, A., Neto de Carvalho, C., Kyriazi, E., . . . Marriner, N. (2008). Principles of Ichnoarchaeology: new frontiers for studying past times. Studi Trentini di Scienze Naturali. Acta Geologica, 83, 43-72

Bass, W. M. (1987). Human osteology. A laboratory and field manual. Columbia, USA: Missouri Arqueological Society.

Bertling, M., Braddy, S. J., Bromley, R., Demathieu, G. R., Genise, J., Mikuláš, R., . . U Uchman, A. (2006). Names for trace fossils: a uniform approach. Lethaia, 39(3), 265-286. doi:10.1080/00241160600787890

Brothwell, D. R. (1987). Desenterrando huesos. La excavación, tratamiento y estudio de restos del esqueleto humano. D.F. México: Fondo de Cultura Económica.

Buckland, P. C. y Coope. G. R. (1991). A bibliography and literature review of quaternary entomology. Dept. of Archaeology \& Prehistory, University of Sheffield. Sheffield: J.R. Collis Publications.

Buikstra, J. E. y Ubelaker, D. H. (1994). Standards for data collection from human skeletal remains, Archaeological Survey Research Series N ${ }^{\circ}$. 44, Arkansas.

Byrd, J. H. y Castner, J. L. (Eds.). (2001). Forensic entomology: The utility of arthropods in legal investigations. Florida, USA: CRC Press.

Constantino, R. (1999). Chave ilustrada para identificação dos gêneros de cupins (Insecta: Isoptera) que ocorrem no Brasil. Papéis Avulsos de Zoologia, 40(25), 387-448.

Derry, D.E. (1911). Damage done to skulls and bones by termites. Nature, 86, 245-246.

Duday, H., Courtaud, P., Crubezy, É., Sellier, P. y Tillier, A.M. (1990). L'Anthropologie «de terrain»: reconnaissance et interprétation des gestes funéraires. Bulletins et Mémoires de la Société d'Anthropologie de Paris, 3-4, 29-49. doi:10.3406/bmsap.1990.1740

Faure, M., Guérin, C. y Luz, M. F. da. (2011). Les parures des sépultures préhistoriques de l'abri-sous-roche d'enoque (Parc National Serra das Confusões, Piauí, Brésil). Anthropozoologica, 46(1), 27-45.

Fontes, L. R. (1995). New genera and new species of Nasutitermitinae from the Neotropical region (Isoptera, Termitidae). Revista Brasileira de Zoologia, 3(1), 7-25.

Frey, R. W. (1973). Concepts in the study of biogenic sedimentary structures. Journal of Sedimentary Petrology, 43,6-19. doi:10.1306/74D726C1-2B21-11D7$8648000102 \mathrm{C} 1865 \mathrm{D}$

Grassé, P. P. (1982). Termitologia: Anatomie, physiologie, biologie systématique des termites. Paris, Francia: Masson.
Gordon, C. C. y Buikstra, J. E. (1981). Soil pH, bone preservation, and sampling bias at mortuary sites. American Antiquity, 46(3), 566-571. doi:10.2307/280601

Guidon, N. y Luz, M. F. (2009). Sepultamentos na Toca do Enoque (Serra das Confusões-Piauí). Fumdhamentos, 8,116123

Huchet, J. B. (1996). L'Archeoentomologie funéraire: une approche originale dans l'interprétation des sépultures. Bulletins et mémoires de la société d'anthropologie de Paris, 8 (3-4), 289-302. doi:10.3406/bmsap.1996.2450

Huchet, J. B. (2010). Archaeoentomological study of the insect remains found within the mummy of Namenkhet Amon (San Lazzaro Armenian Monastery, Venice/ Italy). Advances in Egyptology, 1, 58-80.

Huchet, J. B. (2014a). Approche ichnologique et taphonomique des altérations ostéolytiques dues aux insectes en contexte archéologique. En Denys, C., y Patou-Mathis, M. (Eds.), Manuel de taphonomie, (pp. 185-207). Arles, France: Actes Sud - Errance.

Huchet, J. B. (2014b). Insect remains and their traces: relevant fossil witnesses in the reconstructions of past funerary practices. Anthropologie, 52(3), 329-346.

Huchet, J. B., Deverly, D., Gutierrez, B. y Chauchat, C. (2011). Taphonomic evidence of a human skeleton gnawed by termites in a Moche-civilisation grave at Huaca de la Luna, Peru. International Journal of Osteoarchaeology, 21(1), 92-102. doi:10.1002/oa.1110

Huchet, J. B., Pereira, G., Gomy, Y., Phillips, T. K., Alatorre-Bracamontes, C. E., Vásquez-Bolaños, M. y Mansilla, J. (2013). Archaeoentomological study of a pre-Columbian funerary bundle (mortuary cave of Candelaria, Coahuila, Mexico). Annales de la Société entomologique de France (N.S.), 49(3), $277-$ 290, doi:10.1080/00379271.2013.845474

Kaiser, T. M. (2000). Proposed fossil insect modification to fóssil mammalian bone from Plio- Pleistocene hominidbearing deposits of Laetoli (Northern Tanzania). Annals of the Entomological Society of America, 93(4), 693 700. doi:10.1603/0013-8746(2000)093[0693:PFIMTF] 2.0.CO;2

Kinoshita, A., Sullasi, H. L., Asfora, V. K., Azevedo, R. L., Guzzo, P., Guidon, N., ... Baffa, O. (2016). Dating of fossil human teeth and shells from Toca do Enoque site at Serra das Confusões National Park, Brazil. Anais da Academia Brasileira de Ciências, 88(2), 847855. doi:10.1590/0001-3765201620150083.

Lima, J. T y Costa-Leonardo, A. M. (2007). Recursos alimentares explorados pelos cupins (Insecta: Isoptera). Biota Neotropica, 7(2), 243-250. dói:10.1590/S167606032007000200027.

Luz, M. F. (2014). Práticas funerárias na área arqueológica da Serra da Capivara, sudeste do Piauí, Brasil. (Tesis de Doctorado). Universidade Federal de Pernambuco, Recife, Pernambuco.

Matu, M., Crevecoeur, I. y Huchet, J. B. (2017). Taphonomy and Paleoichnology of Olduvai Hominid 1 (OH1), Tanzania. International Journal of Osteoarchaeology, 27(5), 785-800. doi:10.1002/oa.2593

Matthew, C. (2018). On the significance of insect remains and traces in archaeological interpretation. Global Journal of Archaeology \& Anthropology, 2(4), 555-593. doi:10.19080/GJAA.2018.02.555593

McBrearty, S. (1990). Consider the humble termite: Termites as agents of post-deposition disturbance at African archaeological sites. Journal of Archaeological Science, 17, 111-143. doi:10.1016/0305-4403(90)90054-9

Miller, D.M. (2010). Subterranean Termite Biology and Behavior. Virginia Cooperative Extension; 444-502. 
Recuperado de https://vtechworks.lib.vt.edu/bitstream/ handle/10919/56812/444-502.pdf?sequence $=1$

Moret, P. (1996). Arqueo-entomología: cuando los insectos fósiles contribuyen al conocimiento de nuestro pasado. Boletín de la S.E.A. (Paleoentomología), 16, 183-188.

Nystrom, K. C., Goff, A. y Goff, M. L. (2005). Mortuary behaviour reconstruction through palaeoentomology: a case study from Chachapoya, Perú. International Journal of Osteoarchaeology 15, 175-185. doi:10.1002/ oa. 767

Pessis, A. M. (1992). Identidade e classificação dos registros gráficos pré-históricos do nordeste do Brasil. CLIO Arqueológica, 8(1), 35 - 68.

Prestes, A. C., Tepedino, K. P., Kosmann, C. y Pujol-Luz, J. R. (2014). First record of Rhynchotermes nasutissimus (Silvestri) (Isoptera: Syntermitinae) associated with Rat Carrion in Brasília, Brazil. Entomo Brasilis, 7(1), 5861. Recuperado de https://periodico.ebras.bio.br/ojs/ index.php/ebras/article/view/ebrasilis.v7i1.353

Prestwich, G. D., Bentley, B. D. y Carpenter, E. J. (1980). Nitrogen sources for neotropical nasute termites: fixation and selective foraging. Oecologia, 46, 397-401. doi:10.1007/BF00346270.

Queiroz, R. A., Soriano, E. P., Carvalho, M. V. D., CaldasJunior, A. F., Souza, E. H. A., Coelho-Junior, L.G, ... Vasconcellos, A. (2017). First forensic records of termite activity on non-fossilized human bones in Brazil. Brazilian Journal of Biology, 77(1), 127-131. doi:10.1590/1519-6984.11415

Silva, E. y Andrade, R. M. (2016). Bioturbação por isoptera (arthropoda, hexapoda) em painéis com registros rupestres em sítios arqueológicos inseridos nos roteiros turísticos do Parque Nacional Serra da Capivara, Piauí. CLIO Arqueológica, 31(2), 81-101.
Slaytor, M. y Chappell, D. J. (1994). Nitrogen metabolism in termites. Comparative Biochemistry and Physiology, 107, 1-10. doi:10.1016/0305-0491(94)90218-6

Smith, K. G. V. (1986). A manual of forensic entomology. London, England: Trustees of the British Museum (Natural History).

Sprague, R. (2005). Burial terminology: a guide for researchers. Oxford, England: AltaMira Press.

Thorne, B. L. y Kimsey, R. B. (1983). Attraction of neotropical Nasutitermes termites to carrion. Biotropica, 15(4), 295-296. doi:10.2307/2387656

Ubelaker, D. (1978). Human skeletal remains. Excavation, analysis, interpretation. Washington, USA: Taraxacum.

Vanin, S. y Huchet, J. B. (2017). Forensic entomology and funerary archaeoentomology. En: Schotsmans, E. M., Márquez, G. N., y Forbes, S. L (Eds.), Taphonomy of human remains: forensic analysis of the dead and the depositional environment, (pp. 167-186). New Jersey, USA: John Wiley \& Sons.

Watson, J. A. L. y Abbey, H. M. (1986). The effects of termites (Isoptera) on bone: some archeological implications. Sociobiology, 11, 245-254.

Weiss-Krejci, E. (2011). The formation of mortuary deposits: implications for understanding mortuary behavior of past populations. En: Agarwal, S. C., y Glencross, B. (Eds.), Social bioarchaeology, (pp. 68-106). Blackwell Studies in Global Archaeology, Chichester, England: Wiley-Blackwell.

White, T. D. y Folkens, P. A. (2000). Human osteology, California, USA: Academic Press.

Wood, T. G. (1978). Food and feeding habits of termites. En: Brian, M.V. (Ed.). Production ecology of ants and termites, (pp. 55-80). Cambridge, England: Cambridge University Press. 